\title{
Real-Time Freeway Network Traffic Surveillance: Large-Scale Field-Testing Results in Southern Italy
}

\author{
Yibing Wang, Member, IEEE, Pierluigi Coppola, Athina Tzimitsi, Albert Messmer, \\ Markos Papageorgiou, Fellow, IEEE, and Agostino Nuzzolo
}

\begin{abstract}
This paper reports on some large-scale field-testing results of a real-time freeway network traffic surveillance tool that has recently been developed to enable a number of real-time traffic surveillance tasks. This paper first introduces the related network traffic flow model and the approaches employed to traffic state estimation, traffic state prediction, and incident alarm. The field testing of the tool for these surveillance tasks in the A3 freeway of $100 \mathrm{~km}$ between Naples and Salerno in southern Italy is then reported in some detail. The results obtained are quite satisfactory and promising for further future implementations of the tool.
\end{abstract}

Index Terms-Incident alarm, large-scale field testing, realtime freeway network traffic surveillance, traffic state estimation, traffic state prediction.

\section{INTRODUCTION}

$\mathbf{O}$ NE typical problem that necessitates real-time automatic freeway traffic surveillance is that available real-time measurements may be too much in quantity for manual processing but, in the meantime, are still not sufficient in their spatial coverage to deliver a complete image of real-time traffic conditions for a whole freeway network of interest. More precisely, traffic operators cannot manually handle a flood of real-time spot measurement data that arrive at a frequency of, e.g., every 30-60 s; on the other hand, noisy measurements from a limited number of spot detectors may not suffice to provide the complete traffic information needed, which is particularly the case for congested freeway networks that are sparsely equipped with measurement devices. This condition creates the need for realtime freeway traffic surveillance (see Fig. 1), where available

Manuscript received January 7, 2011; accepted January 16, 2011. Date of publication February 17, 2011; date of current version June 6, 2011. This work was supported in part by the European Commission Information Society Technologies (IST) Program through the project RHYTHM under Grant IST2000-29427, by the Italian Ministry of University and Research through the project PON-SAM under Grant 12897, and by the Engineering New Staff Research Fund of Monash University (2009 and 2010). All statements in this paper are the sole responsibility of the authors and do not necessarily reflect the views and policies of the European Commission, the Italian Ministry of University and Research, and Monash University. The Associate Editor for this paper was F.-Y. Wang.

Y. Wang is with the Department of Civil Engineering, Monash University, Clayton, Melbourne, VIC 3800, Australia (e-mail: yibing.wang@monash.edu).

P. Coppola and A. Nuzzolo are with the Department of Civil Engineering, "Tor Vergata" University of Rome, 00133 Rome, Italy (e-mail: coppola@ ing.uniroma2.it; nuzzolo@ing.uniroma2.it).

A. Tzimitsi and M. Papageorgiou are with the Dynamic Systems and Simulation Laboratory, Technical University of Crete, 73100 Chania, Greece (e-mail: tzimitsi@gmail.com; markos@dssl.tuc.gr).

A. Messmer resides at Groebenseeweg 2, 82402 Seeshaupt, Germany (e-mail: Albert.Messmer@t-online.de).

Color versions of one or more of the figures in this paper are available online at http://ieeexplore.ieee.org.

Digital Object Identifier 10.1109/TITS.2011.2107901

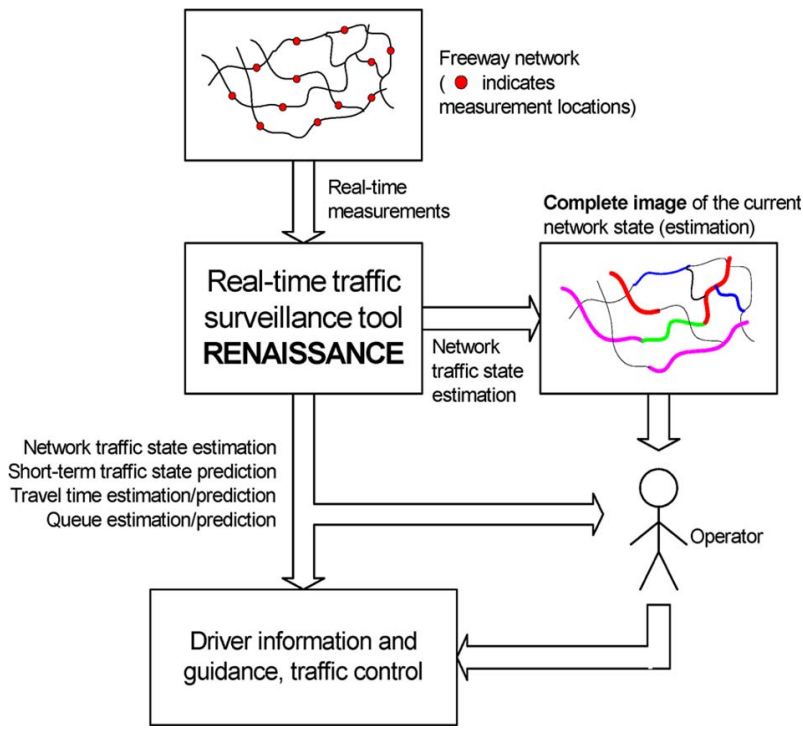

Fig. 1. Real-time freeway network traffic surveillance. [The different colors and widths of links inside the estimated network block represent different traffic conditions (e.g., free flow, dense, and congested conditions) in the corresponding links. Note that, for a practical application, such a global image of the estimated network traffic conditions needs to be updated in real time (e.g., every $1 \mathrm{~min})$.]

raw spot measurement data are appropriately processed, leading to a complete, succinct, and reliable set of information that can be used either for real-time decision making by traffic operators or for further traffic operation (e.g., route guidance and ramp metering). Real-time freeway traffic surveillance involves the following major tasks [1], [2].

- Traffic state estimation refers to estimating traffic flow variables (flows, mean speeds, and densities) for a considered freeway stretch or network, with an adequate spatial resolution (e.g., every $500 \mathrm{~m}$ or less), at each time instant (e.g., every 5-10 s) based on limited measurement data available from traffic detectors (e.g., inductive loops, radar sensors, and video cameras).

- Traffic state prediction refers to predicting traffic flow variables with the same spatial resolution over a futuretime horizon (e.g., 10-20 min), starting at each current time instant.

- Travel time prediction refers to predicting the travel time experienced along any specified network route at each current time instant.

- Incident alarm refers to issuing real-time incident alarms with regard to abnormal events, e.g., traffic accidents and detector faults that occur within the network. 


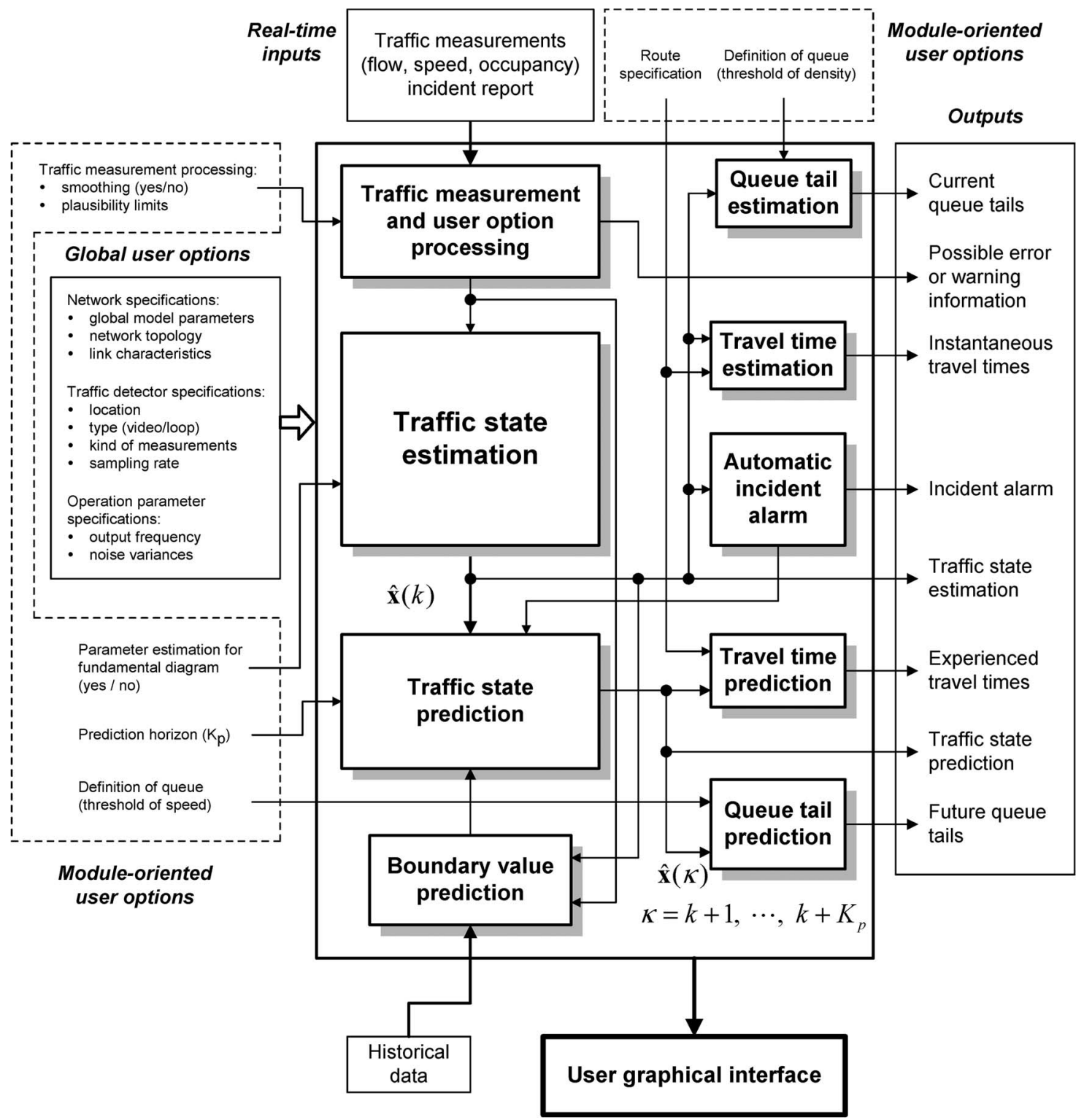

Fig. 2. RENAISSANCE: A real-time freeway network traffic surveillance tool (see also the role of RENAISSANCE in Fig. 1).

One generic real-time freeway network traffic surveillance tool RENAISSANCE [1], [2] (see Fig. 1) has recently been developed, which enables the aforementioned traffic surveillance tasks under a unified methodological framework based on macroscopic traffic flow modeling and extended Kalman filtering (EKF). Fig. 2 depicts the RENAISSANCE functional architecture. RENAISSANCE is currently operational in the southern Italian freeway traffic control center in Naples, Italy, monitoring the $\mathrm{A} 3$ freeway network with a total directed length of $100 \mathrm{~km}$. This paper reports on some of the RENAISSANCE operational results from this site. Floating-car data that reflect real travel times were not available during the test period; therefore, RENAISSANCE's travel time prediction function was not evaluated. Hence, this paper only addresses the tool's traffic state estimation, traffic state prediction, and incident alarm functions, for which a brief literature review is first presented as follows.

The study of freeway traffic state estimation dates back to the early 1970s [3], [4] and has attracted considerable attention in the last 25 years [1]-[16]; see [9] for a concise review and [15] for some further remarks. Following the EKF avenue pursued in most previous work, one general approach to the design of traffic state estimators has recently been developed in [9]. One major innovative feature of this recent work is online model parameter estimation, i.e., real-time joint estimation of traffic flow variables and some key parameters of the traffic flow model employed by the designed traffic state estimator [1], [2], [9]. It is demonstrated in [13], [15], and [16] that online model parameter estimation leads to some significant adaptive capabilities of the designed traffic state estimator. This state estimator has been incorporated into REANISSANCE as one of its major functional modules and has been tested for freeway stretches in simulation [9] and using real data [13], [15], [16] as well as for a freeway network in simulation [1]. Freeway traffic is a large-scale nonlinear dynamic process; in particular, traffic dynamics in freeway networks are much more complex than the traffic dynamics along single freeway stretches. To fully demonstrate the performance of the designed traffic state 
estimator, it is imperative to further evaluate the estimator on a network extent using real data. The field implementation of RENAISSANCE in Southern Italy has provided such a chance.

Technical literature on traffic state prediction is quite vast but usually focuses on traffic flow prediction; see, e.g., [17][22]. One model-based approach to traffic state prediction has recently been developed for RENAISSANCE, which delivers real-time prediction for both flows and mean speeds, and was tested for a hypothetic network in simulation [1]. In this paper, some field prediction results of traffic flows and speeds are presented for the same Italian freeway network.

Incident detection has been one of the central topics of intelligent transportation systems; see [23]-[26] for some recent work. The incident alarm that was considered in this paper is a major attempt toward a type of intelligent incident detection, which is essentially based on online model parameter estimation and can be performed under the aforementioned traffic state estimation framework. In fact, the possibility of performing incident alarms this way was mentioned in [6] without any explicit results presented. Recently, this potential idea has been developed into a promising method for use by RENAISSANCE and was first tested for a German freeway [15], [16]. This paper reports on some field-testing results from southern Italy.

All traffic surveillance tasks that were considered in this paper were conducted based on macroscopic traffic flow modeling and EKF. Considering the suboptimality of the EKF-based solution to the state estimation of nonlinear systems (see a relevant discussion at the end of [1] and [15]), it is challenging to conduct these tasks at a network level. In fact, this paper is the first to report on the field-testing results of these tasks for a large network (100 km in total directed length) that is sparsely equipped with traffic sensors (4-7 km apart).

Section II briefly describes the traffic dynamic system model and the model-based EKF approach to traffic state estimation and prediction, and incident alarm utilized. The field evaluation is reported in the next sections. Some conclusive remarks are given in the final section.

\section{Modeling And Methodology}

\section{A. Dynamic System Modeling for Freeway Network Traffic}

From an engineering point of view, continuous freeway traffic flow may be viewed as a kind of semifluid. A validated second-order macroscopic traffic flow model [27]-[29] can be utilized to describe the motion of traffic flow. The model represents traffic flow dynamics along freeway stretches using aggregate traffic flow variables (e.g., flows, space mean speeds, and densities) through the conservation question, continuity equation, and dynamic-speed equation. In particular, the dynamic-speed equation includes a steady speed-density relationship, from which the fundamental diagram is readily derived [9]. As illustrated in Fig. 3, the fundamental diagram involves the following three important parameters that essentially characterize traffic flow dynamics: 1) free speed; 2) critical density; 3 ) and capacity. To address traffic flow inhomogeneity (reflected in changes of the values of, typically, the free speed and capacity along freeway stretches due to involved curvature,

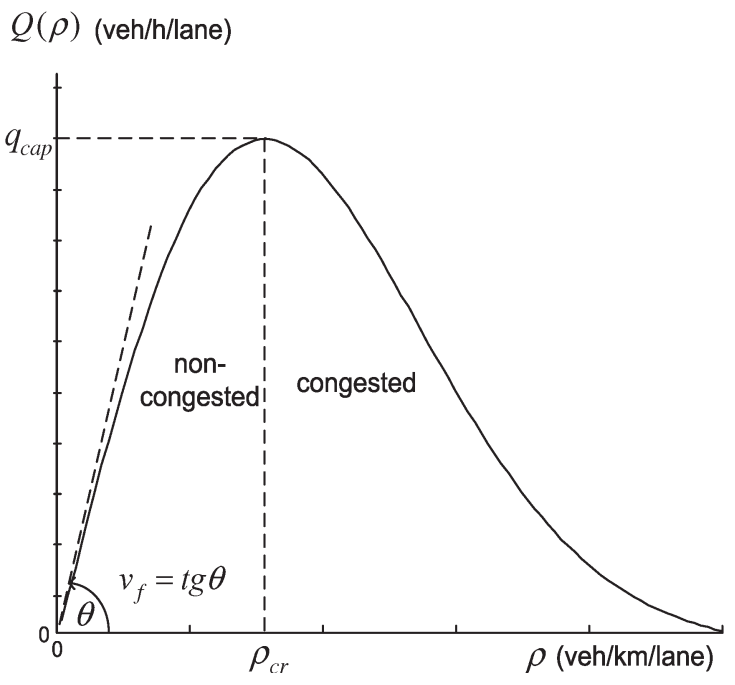

Fig. 3. Qualitative sketch of the fundamental diagram.

slope, tunnels, and speed limits applied), the model may include multiple fundamental diagrams. In addition, the driver behavior at bifurcations is modeled in terms of turning rates. The model is generic in the sense that it can describe traffic flow dynamics in a freeway network of any topology, size, and link characteristics and simulate all kinds of traffic conditions (free flow, dense, and congested), as well as capacity-reducing events (e.g., incidents). For the convenience of modeling and digital computation, the model is presented in a space-time discretized form. For the space discretization, any considered freeway stretch is subdivided into a number of segments, each of $500 \mathrm{~m}$ or less in length, whereas the time discretization is based on a time step (5-10 s) and the discrete time argument $k=$ $(0,1,2, \ldots)$. Thus, aggregate traffic flow variables are defined for each segment and time step. The network model can be expressed in the following compact state-space form:

$$
\boldsymbol{z}(k+1)=\boldsymbol{h}\left[\boldsymbol{z}(k), \boldsymbol{d}(k), \boldsymbol{p}(k), \boldsymbol{\xi}_{\mathbf{1}}(k)\right]
$$

where $\boldsymbol{h}$ is a nonlinear differential vector function that corresponds to all model equations, vector $z$ includes the traffic flow variables (densities and mean speeds of all segments), vector $\boldsymbol{d}$ includes all network boundary variables (mainly inflow at each network origin, e.g., on-ramps, and the turning rate at each bifurcation) [1], [30], vector $\boldsymbol{p}$ includes all fundamental diagram parameters, and vector $\boldsymbol{\xi}_{1}$ includes modeling noise. Considering that $\boldsymbol{d}(k)$ may not fully be measured (or measurable) and $\boldsymbol{p}(k)$ are normally unknown, the following two random-walk equations are introduced:

$$
\boldsymbol{d}(k+1)=\boldsymbol{d}(k)+\boldsymbol{\xi}_{2}(k), \quad \boldsymbol{p}(k+1)=\boldsymbol{p}(k)+\boldsymbol{\xi}_{3}(k) .
$$

Then, the combination of both random-walk equations and (1) leads to the following augmented state-space model:

$$
\boldsymbol{x}(k+1)=\boldsymbol{f}[\boldsymbol{x}(k), \boldsymbol{\xi}(k)]
$$

with traffic state vector $\boldsymbol{x}=\left[\boldsymbol{z}^{\mathrm{T}} \boldsymbol{d}^{\mathrm{T}} \boldsymbol{p}^{\mathrm{T}}\right]^{\mathrm{T}}$ and state noise vector $\boldsymbol{\xi}=\left[\begin{array}{lll}\boldsymbol{\xi}_{1}^{\mathrm{T}} & \boldsymbol{\xi}_{2}^{\mathrm{T}} & \boldsymbol{\xi}_{3}^{\mathrm{T}}\end{array}\right]^{\mathrm{T}}$. 
On the other hand, traffic measurements from any freeway network are related to the traffic state $\boldsymbol{x}(k)$ through the following output equation:

$$
\boldsymbol{y}(k)=\boldsymbol{g}[\boldsymbol{x}(k), \boldsymbol{\eta}(k)]
$$

where vector $\boldsymbol{y}$ consists of all available measurements of flow and mean speed, $\boldsymbol{g}$ is a nonlinear differentiable vector function, and vector $\boldsymbol{\eta}$ is a function of modeling and measurement noise. The state equation (2) and output equation (3) constitute a general dynamic system model for freeway network traffic, upon which, the pursued traffic state estimator and predictor, as well as the incident siren, can be designed. See [1], [9], and [15] for more details of the model.

\section{B. Traffic State Estimation, Traffic State Prediction, and Incident Alarm}

In terms of the model of (2) and (3), traffic state estimation refers to estimating all elements of vector $\boldsymbol{x}$ for a freeway network considered at each time instant $k$ based on limited measurements $\boldsymbol{y}$, whereas the short-term traffic state prediction refers to predicting at each current time instant $k$ all elements of vector $z$ over a future-time horizon (10-20 min). Note that the number of traffic state variables to be estimated and predicted may be much larger than the traffic state variables that were directly measured, particularly when the detectors are sparsely installed within the network.

1) Traffic State Estimation: Because of the strong nonlinearity of the traffic flow model, EKF is used to design the traffic state estimator as

$$
\begin{aligned}
\hat{\boldsymbol{x}}(k+1 \mid k)=\boldsymbol{f}[\hat{\boldsymbol{x}}(k \mid k-1), \mathbf{0}]+\boldsymbol{K}(k) \\
\times[\boldsymbol{y}(k)-\boldsymbol{g}(\hat{\boldsymbol{x}}(k \mid k-1), \mathbf{0})]
\end{aligned}
$$

where $\hat{\boldsymbol{x}}(k+1 \mid k)$ denotes the traffic state estimation for time instant $k+1$ based on traffic measurements $\boldsymbol{y}(k)$, and $\boldsymbol{K}(k)$ is the gain matrix and is calculated online based on the linear Taylor expansion of $\boldsymbol{f}$ and $\boldsymbol{g}$ at $\hat{\boldsymbol{x}}(k \mid k-1)$. For simplicity, we will denote $\hat{\boldsymbol{x}}(k \mid k-1)$ by $\hat{\boldsymbol{x}}(k)$ in the following discussion. Keeping in mind the constitution of vector $x$, (4) simultaneously delivers, in real time, the estimates of traffic flow variables with the estimates of the fundamental diagram parameters, enabling the so-called online model parameter estimation [1], [2], [9], [13], [15], [16]. Because the model may include multiple fundamental diagrams to address the aforementioned traffic flow inhomogeneity, the estimator (if supported with sufficient measurements) can deliver the parameter estimates for each fundamental diagram.

2) Incident Alarm: Incident alarm, under the aforementioned methodological framework, is essentially based on online model parameter estimation and can therefore be regarded as an extension of traffic state estimation. The traffic flow model that was utilized largely represents the specific and prevailing traffic flow characteristics through the free speeds, critical densities, and capacities. In the presence of incidents, the local traffic flow characteristics may abruptly and substantially change, and such changes can be reflected in drastic real-

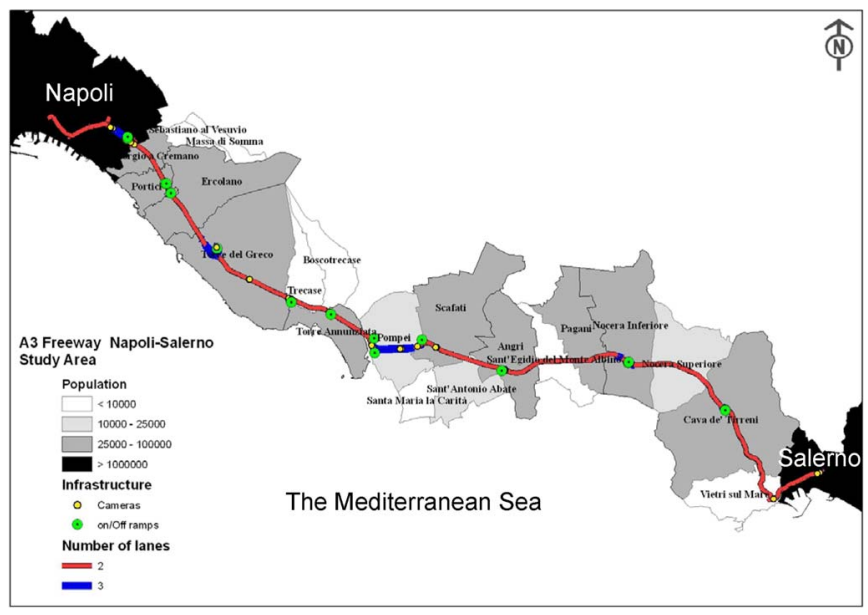

Fig. 4. A3 freeway between Naples and Salerno in southern Italy.

time changes of the model parameter values. Within the EKFbased traffic state estimation framework, traffic measurement that reflects local traffic flow conditions is sequentially fed to the operational traffic state estimator. Thus, through online model parameter estimation, the resulting changes of the local parameter values can be identified and tracked in real time, thereby leading to automatic incident alarm for traffic operators. See [15] and [16] for some illustrative incident-alarm examples, and one more example is presented in this paper.

3) Traffic State Prediction: Neglecting unpredictable random noise $\boldsymbol{\xi}_{1}$, (1) reads

$$
\boldsymbol{z}(k+1)=\boldsymbol{h}[\boldsymbol{z}(k), \boldsymbol{d}(k), \boldsymbol{p}(k), \mathbf{0}] .
$$

Let $\hat{\boldsymbol{z}}(k), \hat{\boldsymbol{d}}(k)$, and $\hat{\boldsymbol{p}}(k)$ denote, respectively, the estimates of $\boldsymbol{z}(k), \boldsymbol{d}(k)$, and $\boldsymbol{p}(k)$ at the current time instant $k$. Let $\hat{\boldsymbol{d}}(\kappa)$, $\kappa=k+1, k+2, \ldots, k+K_{p}-1$ denote the prediction of $\boldsymbol{d}$ over the future time horizon of $K_{p}-1$ steps. Note that, for each $k, \hat{z}(k)$, the following conditions hold.

- $\hat{\boldsymbol{d}}(\mathrm{k})$ and $\hat{\boldsymbol{p}}(k)$ are available from $\hat{\boldsymbol{x}}(k)$ through the traffic state estimator (4).

- The model parameters are normally not rapidly time varying; therefore, $\hat{\boldsymbol{p}}(\kappa)$ may be set equal to $\hat{\boldsymbol{p}}(k)$ for $\kappa=$ $k+1, \ldots, k+K_{p}-1$.

- $\hat{\boldsymbol{d}}(\kappa)$ is available for $\kappa=k+1, \ldots, k+K_{p}-1$ through boundary value prediction.

Thus, running (5) for $K_{p}$ steps produces $\hat{\boldsymbol{z}}(\kappa), \kappa=k+$ $1, \ldots, k+K_{p}$, which is the pursued traffic state prediction. The boundary value prediction is indispensable to traffic state prediction and can be performed based on historical data, through the extrapolation of the available boundary variable estimates, or by an appropriate combination of both [1], [30].

\section{LaRGE-Scale Test Site in South Italy}

\section{A. Site Description}

The designed traffic state estimator and predictor, as well as the incident siren, along with the traffic surveillance tool 


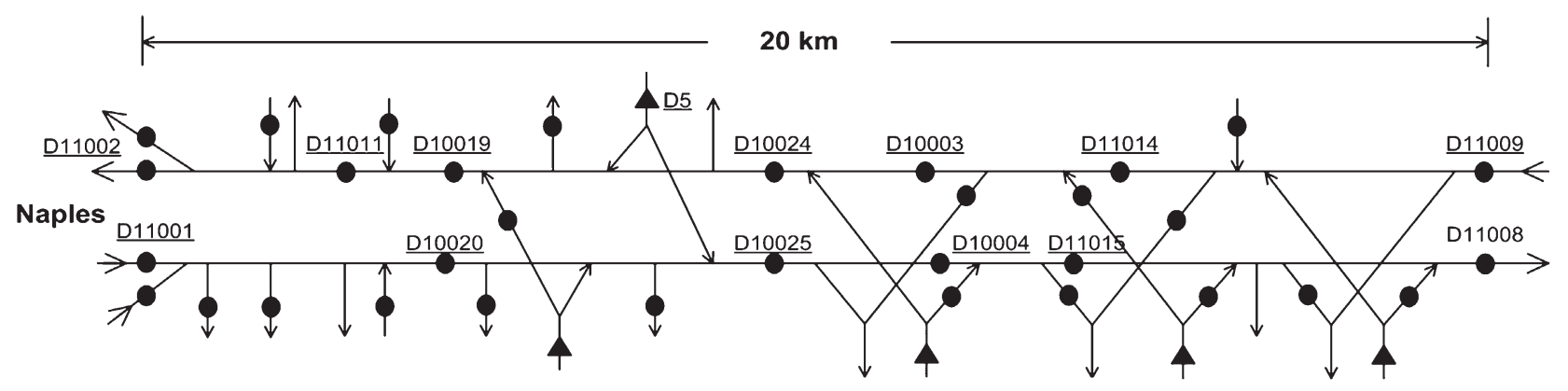

(a)

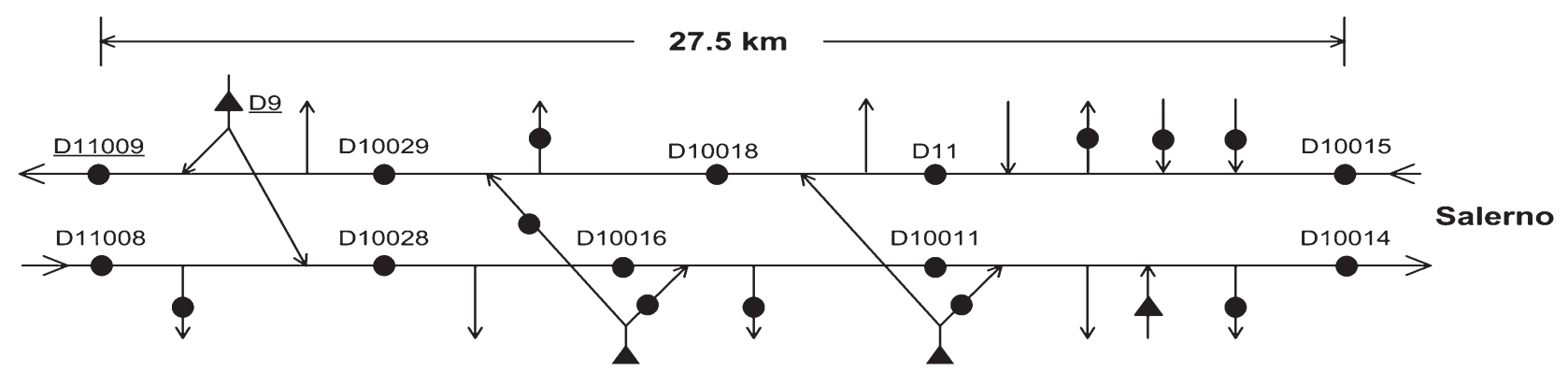

(b)

Fig. 5. Detector configuration in the A3 freeway (with each dot representing a video sensor and each triangle representing a toll station).

REANAISSANCE, are currently operational at the south Italian freeway traffic control center in Naples, supervising the A3 freeway between Naples and Salerno with a total directed length of about $100 \mathrm{~km}$ (see Fig. 4). The A3 freeway connects Naples and Salerno with the municipalities of the coastal strip along the southern slope of Mount Vesuvius. In the last decades, these municipalities have considerably grown despite the limited space between the coast and Vesuvius. This narrow area is currently among the most densely populated regions in Italy. Because most of the population in these municipalities commutes daily toward/from Naples, the A3 freeway serves one of the most congested metropolitan areas in southern Italy. As a consequence, recurrent congestions occur in the A3 freeway. In the outlined traffic context, the operation of RENAISSANCE is expected to considerably enhance the traffic surveillance capability of the freeway traffic control center in Naples.

Fig. 5 presents the layout of the A3 site and its detector configuration in both directions, where each black dot represents a video detector that offers flow and speed measurements, whereas each black triangle just upstream each pair of onramps represents a toll station that records only the number of passing vehicles. The detectors are quite sparsely installed in the mainstream of either direction, with an average spacing of $4 \mathrm{~km}$ for the 20-km section at the Naples side [see Fig. 5(a)] and an average spacing of $6.9 \mathrm{~km}$ for the other $27.5-\mathrm{km}$ section at the Salerno side [see Fig. 5(b)].

\section{B. Analysis of Measurement Data}

Fig. 6(a) and (b) displays 24-h speed measurements that were collected in the Salerno and Naples directions, respectively, on May 25, 2006. As shown, free-flow conditions prevail for the whole day in the Salerno direction, except for some temporary events that cause local speed drops around 9:00 P.M. However, sharp speed drops in the Naples direction were recorded that morning at detectors D10019, D10024, D10003, and D11014 [see these detectors in Fig. 5(a)]. Zooming on Fig. 6(b) for the congested period, we more clearly see in Fig. 6(c) that the congestion shockwaves sweep the stretch, including D10019, D10024, D10003, and D11014, during 7:30-10:30 A.M. but dissolved before reaching D11009. Moreover, it is also shown that the speed-drop pattern that was observed at D10019 is quite different from the pattern that was observed at the upstream detectors D10024, D10003, and D11014. This case may partially be because of the impact of the complex site layout (and related ramp flows between D10019 and D10024) on the shockwave propagation. In addition, we can see a clear distinction between the speed measurements under free-flow conditions [compare, e.g., the measurements of D11014 and D11009 in Fig. 6(c)].

\section{Site Modeling}

This site involves several internal bifurcations, each at the immediate downstream of a toll station, e.g., right below D5 in Fig. 5(a); hence, the site is essentially a freeway network. For traffic state estimation, both directions of the freeway have to be considered all together, because the sparse detector installation in the mainstream does not provide sufficient measurements for the two directions to separately be treated (for traffic state estimation). More specifically, due to measurement insufficiency, only if the site is considered as a network (rather than two individual stretches in the opposite directions) can the system observability be guaranteed, and hence, the traffic state estimation task is feasible [1].

Without involving obvious geometrical homogeneity (e.g., lane drop or significant curvature/slope change), any freeway 




(a)

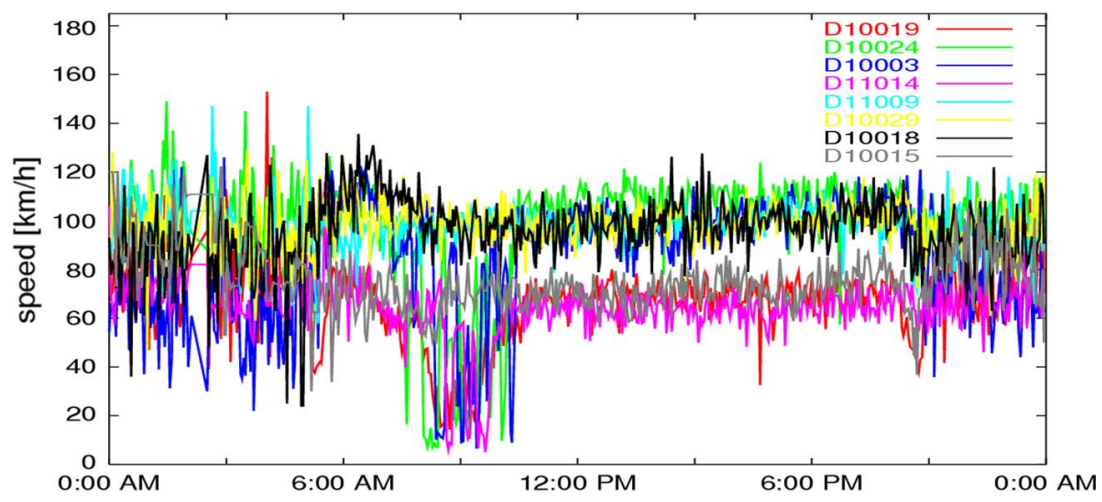

(b)

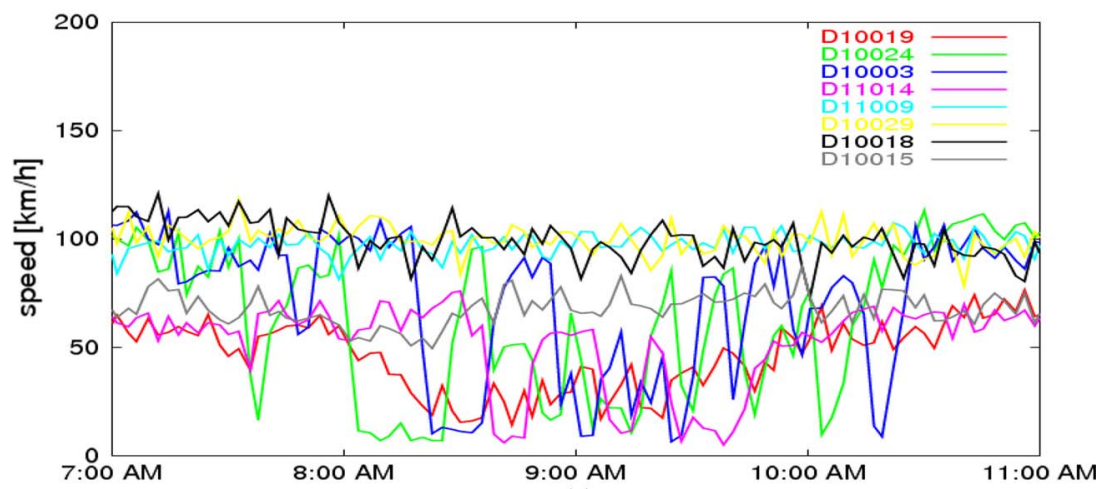

(c)

Fig. 6. Speed measurements on May 25, 2006. (a) Salerno direction. (b) Naples direction. (c) Zoom-on image of (b).

stretch in Fig. 5 between a pair of neighboring on/off-ramps is defined as a link. Every link is subdivided into a number of segments, each of about $500 \mathrm{~m}$. All links and segments of such normally follow the same fundamental diagram. The interested reader is referred to [1] for more details on the definitions of links and segments. As aforementioned, however, every day, significant differences in the mainstream speed measurements can be observed under free-flow conditions [see Fig. 6(b) and (c)]. Such spatial speed variation is normally due to traffic flow heterogeneity, i.e., the free-speed value may change over distance due to curvature involved, slope, tunnels, and speed limits applied. To adequately address such traffic flow inhomogeneity, multiple fundamental diagrams need to be introduced to the utilized traffic flow model equation (1), each with a separate group of free speed, critical density, and capacity, addressing a specific directional stretch between two adjacent mainstream detectors. Thus, 17 fundamental diagrams were considered for this test example, and the dimension of vector $\boldsymbol{p}$ in (1) is 51 . Overall, the number of traffic state variables to be estimated (i.e., the rank of vector $\boldsymbol{x}$ ) is more than 500 , whereas the detectors and toll stations deliver measurements for 59 flow variables and 46 speed variables only. In other words, the majority of state variables of interest have to be estimated from very limited measurement data.

\section{TRAfFic State Estimation Results}

Flow and speed measurements that were collected from all detectors and toll stations at the site on May 25, 2006, were considered for testing. The update interval of the utilized measurements is irregular but about $30 \mathrm{~s}$, on average, whereas the model time step was set equal to $5 \mathrm{~s}$. Some representative traffic state estimation results of RENAISSANCE are presented in Figs. 7-9. 

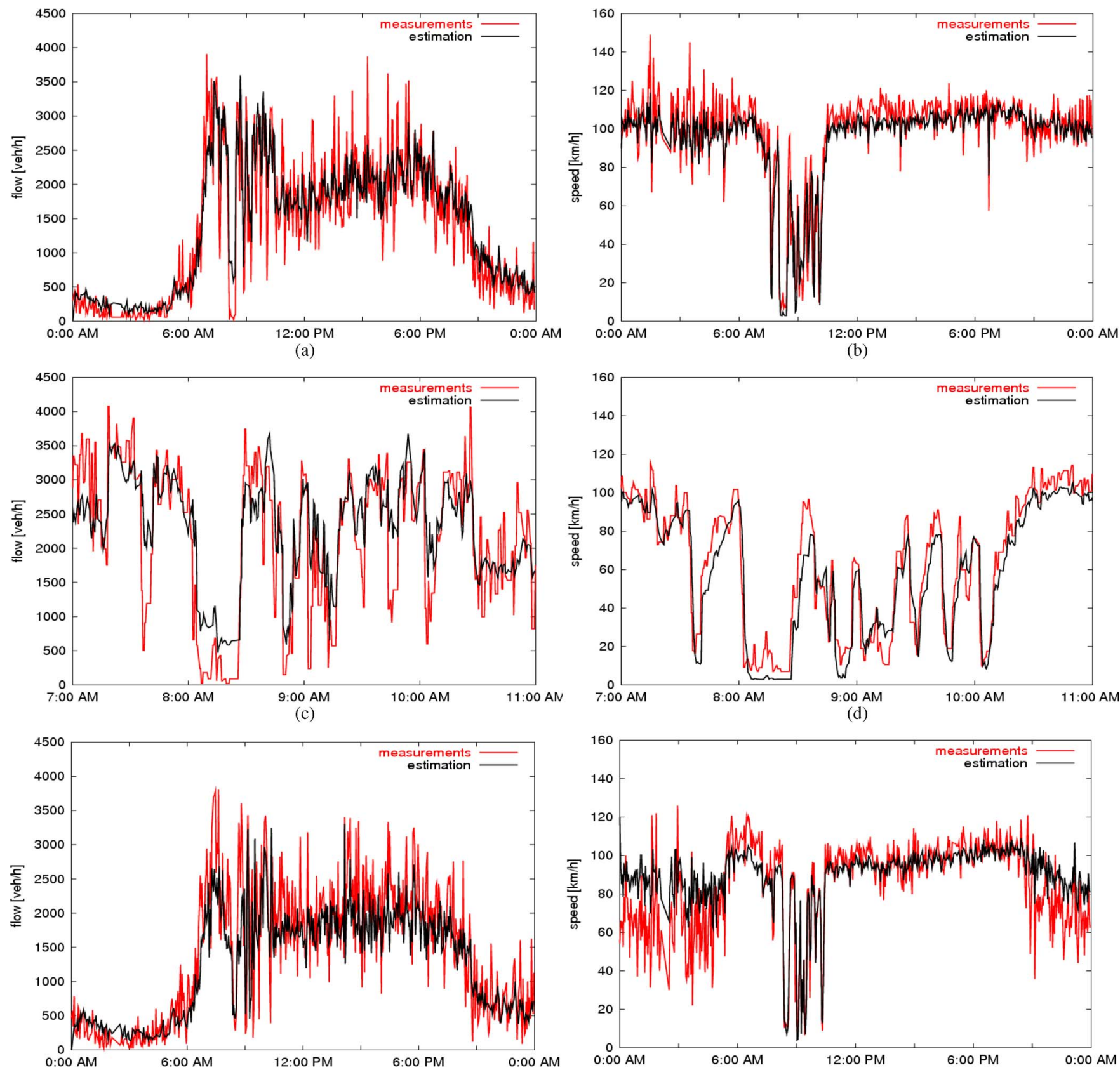

(e)

(f)
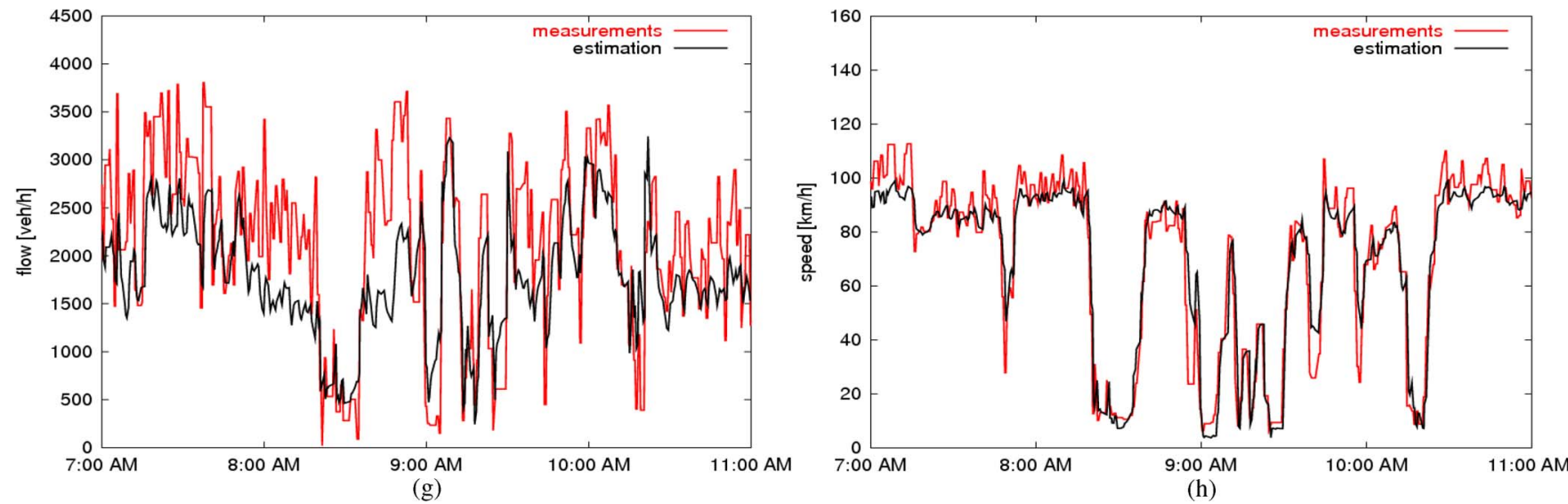

Fig. 7. Traffic state estimates in the Naples direction on May 25, 2006. (a) and (b) At D10024. (c) and (d) Zooming on (a) and (b). (e) and (f) At D10003. (g) and (h) Zooming on (e) and (f). 

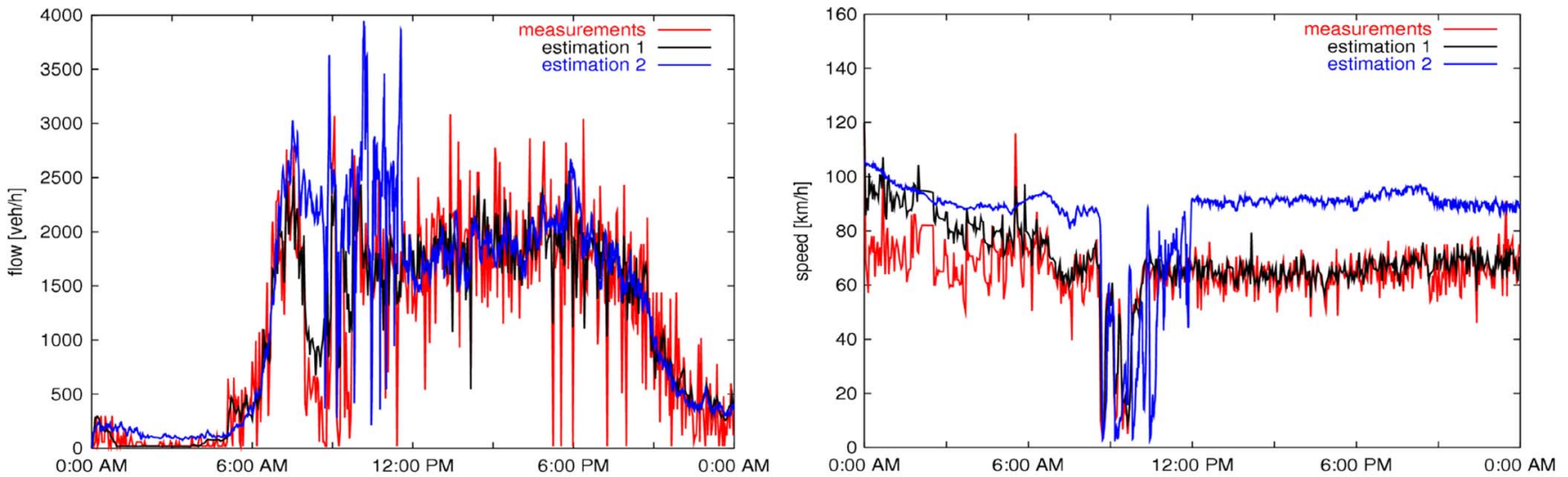

(a)

(b)
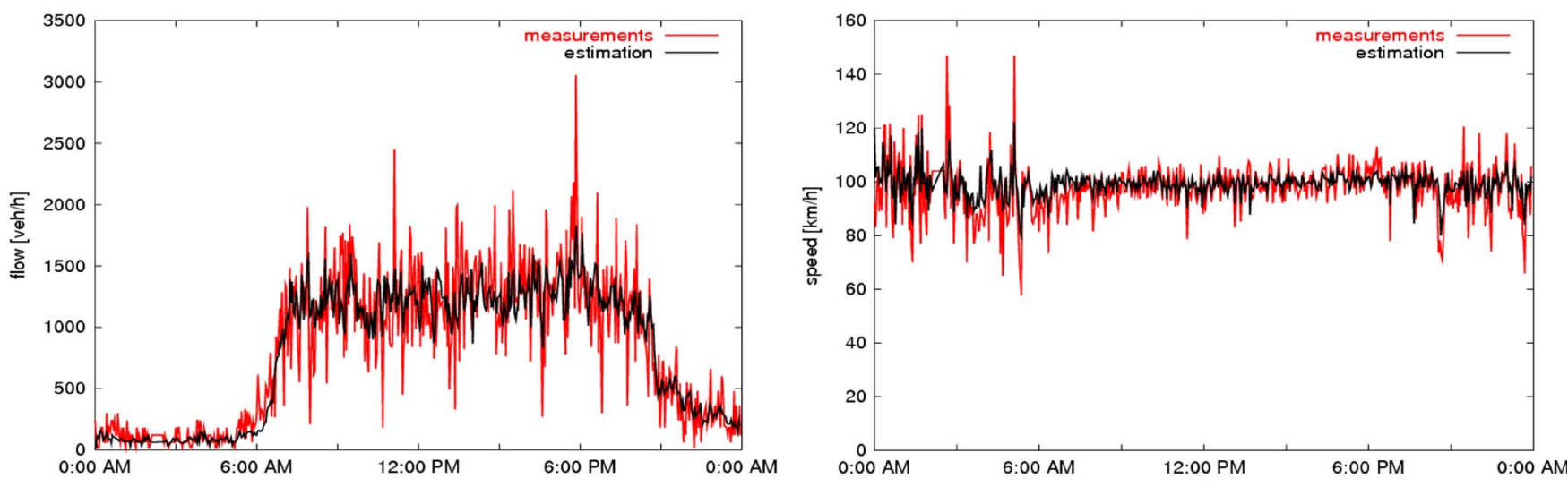

(c)


(e)

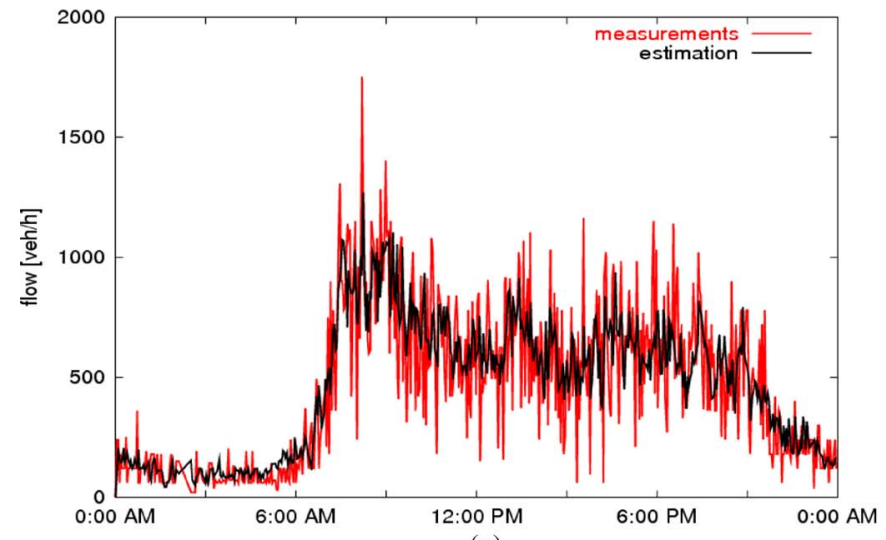

(g)

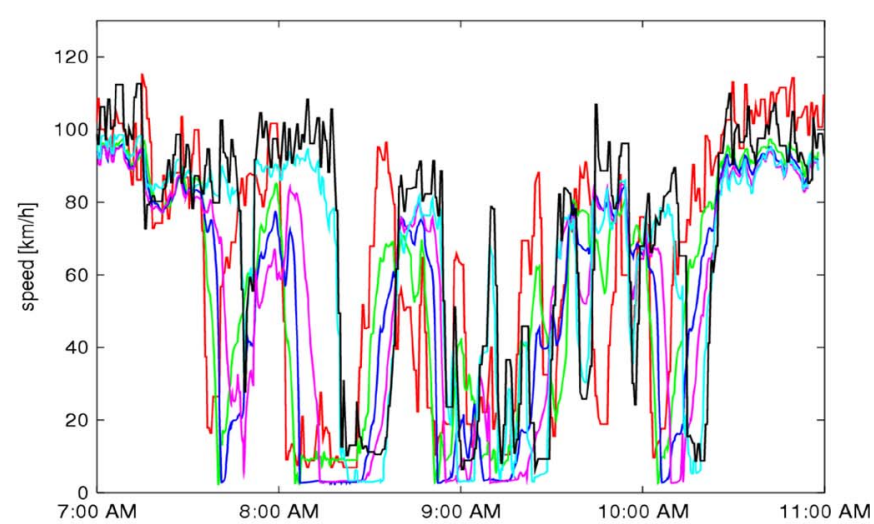

(h)

Fig. 8. Traffic state estimates in the Naples direction on May 25, 2006. (a) and (b) At D11014. (c) and (d) At D11009. (e) and (f) Free-speed estimates. (g) At D5. (h) Speed estimates along the stretch between D10024 and D10003. 
(a)



(b)



(c)

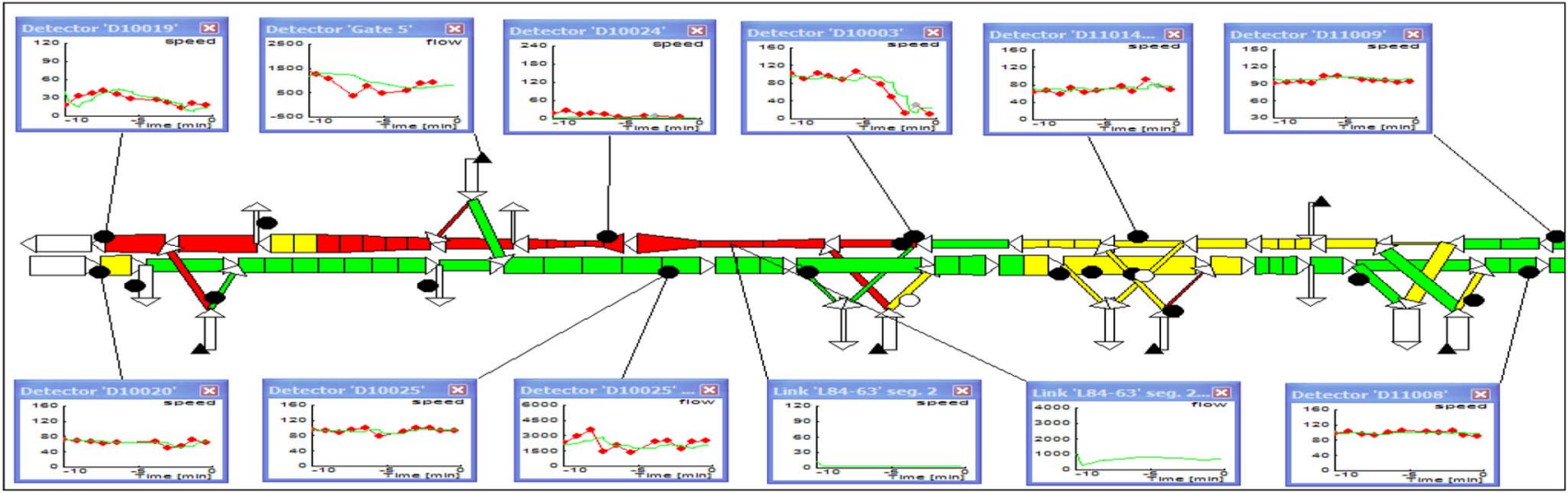

(d)

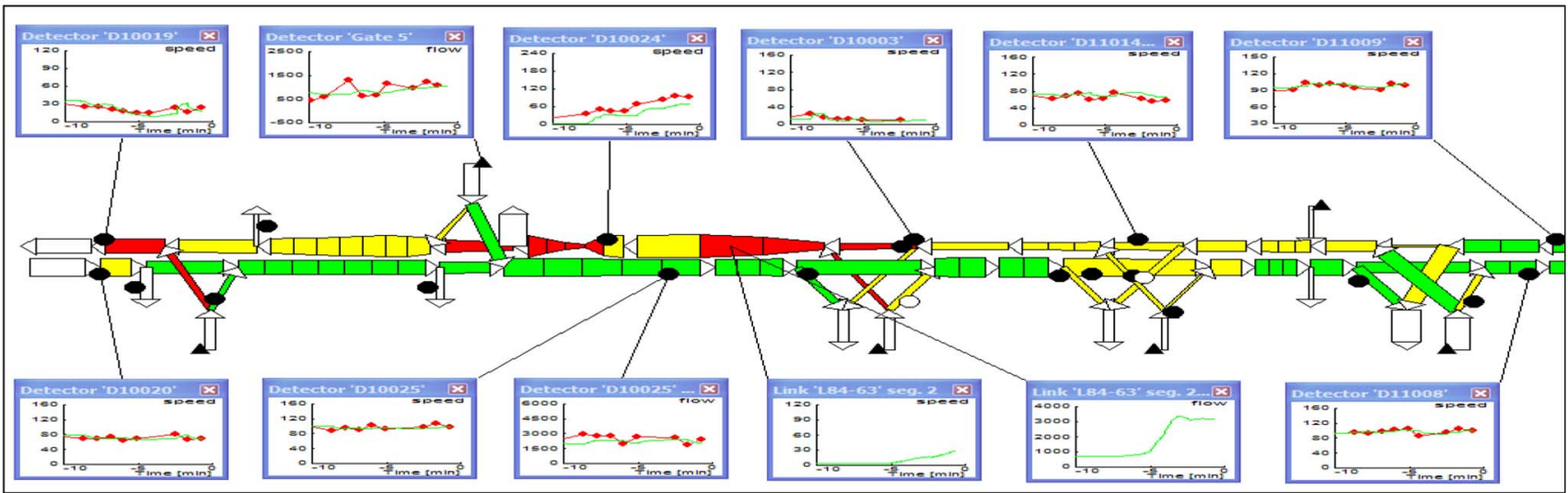

Fig. 9. Real-time estimated complete image of traffic conditions in the A3 freeway during 7:37-8:33 A.M. on May 25, 2006 (from top to bottom, each subfigure corresponds to a different time moment; "green" refers to the segment speeds above $90 \mathrm{~km} / \mathrm{h}$, "red" refers to the segment speeds below $40 \mathrm{~km} / \mathrm{h}$, and "yellow" means otherwise, with the width of each segment corresponding to the segment flow). 


\section{A. Spot Traffic State Estimation Results}

1) Traffic State Estimates at Measurement Locations: The traffic state estimator delivers estimates for all network traffic flow variables (i.e. flow, density, and mean speed of nearly every $500 \mathrm{~m}$ ), including the estimates that were measured (at the detector locations) and unmeasured. The estimation results at some detector locations are presented first. Fig. 7(a) and (b) compares flow and speed estimates with the corresponding measurements at D10024, and Fig. 7(c) and (d) zooms, respectively, on Fig. 7(a) and (b) for the stop-and-go time period. Fig. 7(e)-(h) presents the same comparison and zooming for D10003. The estimates track down the corresponding measurements well at both detector locations. Fig. 8(a) and (b) compares the flow and speed estimates ("estimation 1") with the corresponding measurements at D11014, and Fig. 8(c) and (d) delivers the same comparison at D11009. Note that all these estimation results were obtained by considering multiple fundamental diagrams to address the apparent traffic flow inhomogeneity. Fig. 8(e) and (f) displays the free-speed estimates for the stretches bounded downstream by D11014 and D11009, respectively. In fact, if the observed traffic flow inhomogeneity is ignored (in which case only one fundamental diagram is considered for the whole A3 network), striking estimation bias appears at D11014 [see "estimation 2" in Fig. 8(a) and (b)]. This occurs because the speed measurements at D11014 are substantially lower than the measurements from most of the other detectors [see Fig. 6(b)], and the estimate of the sole free speed that was defined for the whole network tends to follow the "typical" speed level, giving rise to the estimation bias at D11014. See [30] and [31] for more estimation results that were obtained with the traffic flow inhomogeneity ignored. The flow estimates at the toll station D5 is presented in Fig. 8(g). In terms of network modeling, the flow at D5 is a boundary variable [1], and its estimation is the sum of the estimates of unmeasured flows at the two downstream on-ramps.

Although not presented due to space limits, the flow and speed estimates well match the corresponding measurements at all other detector locations.

2) Traffic State Estimates at Nonmeasuring Locations: As aforementioned, the traffic state estimates at all measurement locations are quite satisfactory, but this result is not yet sufficient for full verification of the capability of the designed traffic state estimator, because the estimates that were produced refer to measurement data that were actually used by the estimator. ${ }^{1}$ Therefore, of particular importance to the performance evaluation is the plausibility of flow and speed estimation at any locations where no measurements were available. Fig. 8(h) plots speed estimates for the Naples-bound stretch between D10024 and D10003 (2.5 km apart), where we have a red curve for D10024 and a black curve for D10003, whereas

\footnotetext{
${ }^{1}$ Nevertheless, because the estimates that were derived from (4) are not simply a duplicate of the corresponding measurement [the second term of (4)], the good matching between estimates and measurements in Figs. 7 and 8 indicates the compatibility of the employed model (the first term of the equation) with the measurement data utilized.
}

any other curve addresses a certain segment between D10024 and D10003. A plausible realistic shockwave that propagates sequence can be observed in the figure; more precisely, the following rule can be used to determine the plausibility and correctness of the estimated shockwave propagation:

Rule 1: When a congestion shockwave propagates upstream, the estimated speed drop at any segment between two neighboring detectors, e.g., the sequential segments that correspond to the green, blue, pink, and light blue curves in Fig. 8(h), should not appear before the congestion reaches the downstream detector (the red curve for D10024) and should not appear later than the speed drop that was already observed at the upstream detector (the black curve for D10003).

Because the A3 freeway was sparsely installed with traffic detectors in its mainstream, it was imperative to feed RENAISSANCE with all available measurements to get the presented results. Whenever possible, however, some redundant measurements should exclusively be used for evaluation, in which case, such measurements are not fed to RENAISSANCE. In fact, the traffic state estimator of RENAISSANCE was extensively tested this way through simulation [1], [9] and using real data [13], [15], [16] based on other (smaller) test examples.

\section{B. Temporal-Spatial Traffic State Estimation Results}

1) GUI of RENAISSANCE: RENEAISSANCE is accompanied with a dedicated graphical user interface (GUI). This GUI can be used for a complete presentation of real-time traffic state estimation results. ${ }^{2}$ Four screenshots of the GUI view that was taken between 7:00 A.M. and 9:00 A.M. are presented in Fig. 9, all focusing on the same A3 section between D10019 and D11009. In real-time operation, the GUI view is updated at the frequency of measurement update ( $30 \mathrm{~s}$ to $1 \mathrm{~min}$ ). Within a GUI screen, each link (headed by an arrow) is displayed along with its segments, each with a length of approximately $500 \mathrm{~m}$. The width of each segment is proportional to the estimated segment flow, whereas the colors of each segment correspond to the following estimated speed levels:

1) green for free-flow conditions, with the segment's space mean speed more than $90 \mathrm{~km} / \mathrm{h}$;

2) yellow for dense flow conditions, with the segment's space mean speed between $90 \mathrm{~km} / \mathrm{h}$ and $40 \mathrm{~km} / \mathrm{h}$;

3 ) red for congested conditions, with the segment's space mean speed less than $40 \mathrm{~km} / \mathrm{h}$.

These threshold values are user dependent. With the same convention used in Fig. 5, any black node in Fig. 9 represents a video camera, whereas a triangle stands for a toll station. By clicking on any black node, triangle, or segment, a diagram window pops up; for a measurement location, both measurements (in red) and estimates (in green) are displayed in the window, whereas for a nonmeasurement location, only estimates are displayed. Each diagram display can be switched between flow, speed, and density, except for toll-charging locations (where the speed values are of no interest). Moreover, each diagram

\footnotetext{
${ }^{2}$ Recall the complete image shown in Fig. 1.
} 
window displays the corresponding estimates (and measurements) over a configurable (past) time period until the current time instant.

Each of the four subfigures in Fig. 9 addresses the A3 network portion, including D10019, D10024, D10003, D11014, and D11009, which is of particular interest because of the dominating stop-and-go traffic behavior that is typically observed there in the morning. It should, however, be mentioned that the GUI can simply be panned over to see any other part of the network.

2) Complete Temporal-Spatial Image of Estimated Traffic Condition: As shown in Fig. 9(a), the displayed freeway network is under free-flow or dense conditions at 7:37 A.M., except for a congestion that mounts at D10019. The congestion shockwave that was estimated keeps propagating upstream with growing strength and reaches D10024 at 8:04 A.M. [see Fig. 9(b)], at which time, one segment upstream of D10024 has already turned dense (because of the impact of downstream density). At 8:22 A.M., the shockwave front of the same congestion (further strengthened because of on/off-ramps encountered during its propagation) reaches D10003 [see Fig. 9(c)]. At 8:33 A.M., with the downstream flow increasing (as indicated by the width of the off-ramp immediately downstream of D10024), the congestion starts to dissolve at D10024 [see Fig. 9(d)]. It is shown in Figs. 6-8 that oscillatory stop-andgo waves dominate in the stretch between D10019 and D11014 that morning. As observed from the GUI, the propagating and dissipating processes of shockwaves that are similar to the processes presented in Fig. 9 repeat until 10:40 A.M.; quite often, a congestion shockwave propagates upstream to join a preceding one. Finally, the last congestion was dissolved around 10:40 A.M. As shown in Figs. 6 and 8 and also confirmed with the estimated temporal-spatial images in Fig. 9, that morning, no shockwave reached D11009 and further upstream.

At this point, we are concerned with how we can evaluate the correctness of the estimated global temporal-spatial images (e.g., the images in Fig. 9). Because no independent measurements were available for the evaluation, we check whether the estimated shockwave propagation reasonably well matches the speed measurements at the detectors that the shockwaves encounter while propagating upstream. As displayed in Fig. 9(b), the moment that the estimated shockwave front (i.e., the uppermost boundary of the segments in red) reaches D10024 is exactly the moment that the speed drop was, indeed, observed by D100024 (see the popped-up diagram at D10024 and the displayed measurement estimation matching, as well as the location of the shockwave front). The same temporal-spatial consistency between the estimated global image and spot measurements is also shown for D10003 and D10024 in Fig. 9(c). In addition, the estimated shockwave dissipation around D10024 in Fig. 9(d) is also shown to be consistent with the measured speed increase. Again, the observed congestion discharging was relevant to the increase of the off-ramp flow right at the upstream of D10024.

In summary, the following rule can be used to assess the correctness of the estimated temporal-spatial complete images:

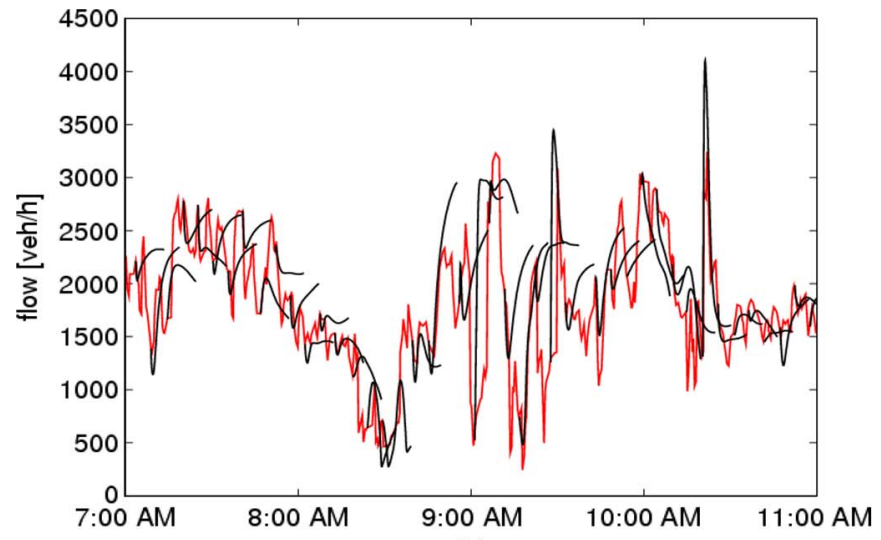

(a)

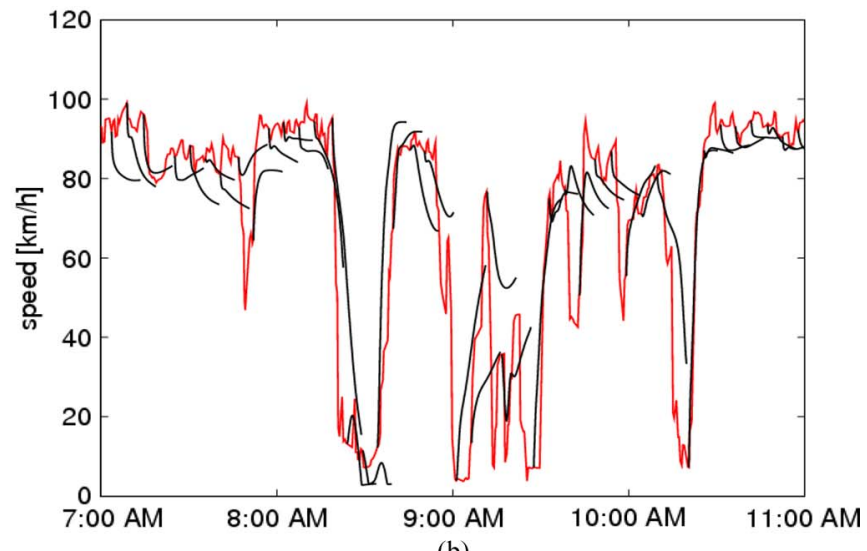

(b)

Fig. 10. Traffic state prediction at D10003 on May 25, 2006. (a) Flow. (b) Speed.

Rule 2: A measured speed drop should be visible by a certain detector approximately at the same time as the estimated congestion shockwave in the temporal-spatial image reaches the detector location; on the other hand, if congestion is measured to be dissolving at a certain measurement location, this case should also accordingly be reflected in the estimated temporal-spatial image, and vice versa.

Based on rules 1 and 2, it may be concluded that the estimation results in Figs. 8(h) and 9 reliably reflect the real traffic conditions and, therefore, the designed traffic state estimator delivered satisfactory traffic state estimates for this largescale site.

The GUI presents, at each time moment, a spatially global view for the whole freeway network of interest (see also Fig. 1), whereas Figs. 7 and 8 deliver, for each detector location, a temporally global view (more than $24 \mathrm{~h}$ ). The former case is more suitable for real-time use in traffic control centers, whereas the latter case is more convenient for detailed offline evaluation.

\section{TRAFFic State Prediction AND INCIDENT ALARM RESULTS}

As shown with (5), the model-based traffic state predictor delivers real-time prediction of traffic flow variables for each segment. The traffic state predictor was also tested for the 




(a)



(b)

Fig. 11. Speed measurements on June 10, 2006. (a) In the Salerno direction. (b) In the Naples direction.

A3 freeway using the measurement data from the same day. The flow and speed prediction results at D10003 are displayed in Fig. 10. Because the measurement estimate comparison at the same detector is already presented in Fig. 7(g) and (h), Fig. 10(a) compares the flow prediction with flow estimate at D10003, and Fig. 10(b) compares the speed prediction with speed estimate at D10003. Note that the red curves in Fig. 10 represent the estimation results, i.e., the black curves in Fig. 7(g) and (h), whereas each short black trajectory represents a prediction of $10 \mathrm{~min}$, starting from the corresponding (current) traffic state estimate. The prediction results are shown to forecast the evolving tendency of the flow and speed dynamics at D10003.

Fig. 11 presents the speed measurements of June 10, 2006, whereas the corresponding traffic flow and speed estimates at D11014 and D11009 are displayed in Fig. 12(a)-(d). As shown in Fig. 11(b), a sharp speed drop was recorded only by D11014 that morning, whereas a sharp flow drop was observed only at D11014 [see Fig. 12(a)]. This case indicates the occurrence of a local incident between D10003 and D11014. Although not presented, an expected flow drop is hardly shown at D10003, and this condition could be because the incident occurred somewhere close to D11014 under the circumstance of the presence of on/off-ramp between D10003 and D11014. Fed with these measurement data, the state estimator delivers satisfactory flow and speed estimates for the whole network (except for the flow at D11014). Moreover, the capacity estimates for the local stretches that were downstream bounded by D11014 and D11009 are presented in Fig. 12(e) and (f), respectively, and the corresponding capacity derivatives are plotted in Fig. 12(g) and (h). Note that the spikes shown in Fig. $12(\mathrm{~g})$ can be used as a base for incident detection. It is interesting to compare the consistency between these incident alarm results and the results in [15] and [16], although for different sites.

The state prediction and incident alarm results that were obtained are deemed satisfactory and promising. These surveillance functions of RENAISSANCE will undergo further evaluation, particularly with respect to, e.g., the duration of feasible prediction time horizons and false-alarm rate.

\section{CONCLUSION}

A freeway network is a large-scale nonlinear dynamic system, and real-time network traffic surveillance is a very challenging task. More specifically, there are performance uncertainties before a network traffic surveillance tool is sufficiently tested in field. This paper has reported on a largescale Italian field evaluation of the traffic state estimator, traffic state predictor, and incident siren of the traffic surveillance tool RENAISSANCE, which has recently been developed. The evaluation results are quite satisfactory. To the best of our knowledge, this paper is the first to report on the field evaluation of real-time traffic surveillance for such a large freeway network. For the reported traffic state estimation task, RENAISSANCE took a total of $11 \mathrm{~h}$ to handle all available measurement data of $24 \mathrm{~h}$. This result means that, assuming that measurement data were updated every $1 \mathrm{~min}$, RENAISSANCE would need approximately $30 \mathrm{~s}$ to do its state estimation job and then stay idle until the next arrival of measurement data. Therefore, 




(a)



(c)

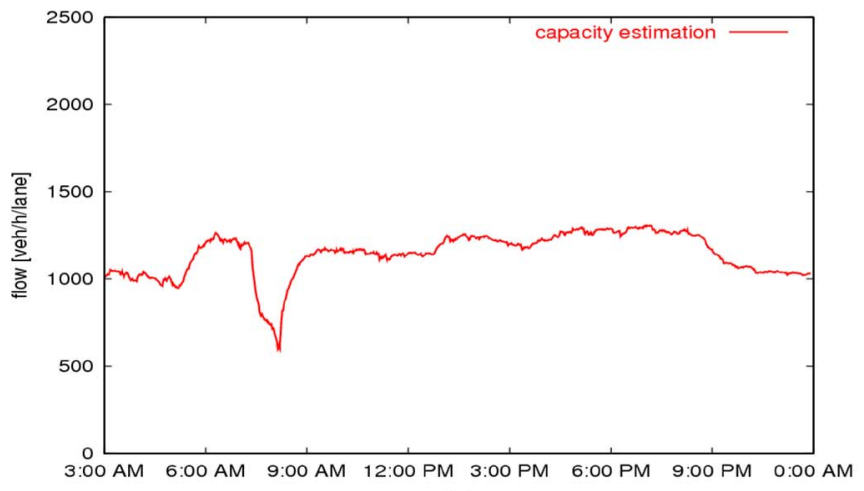

(e)

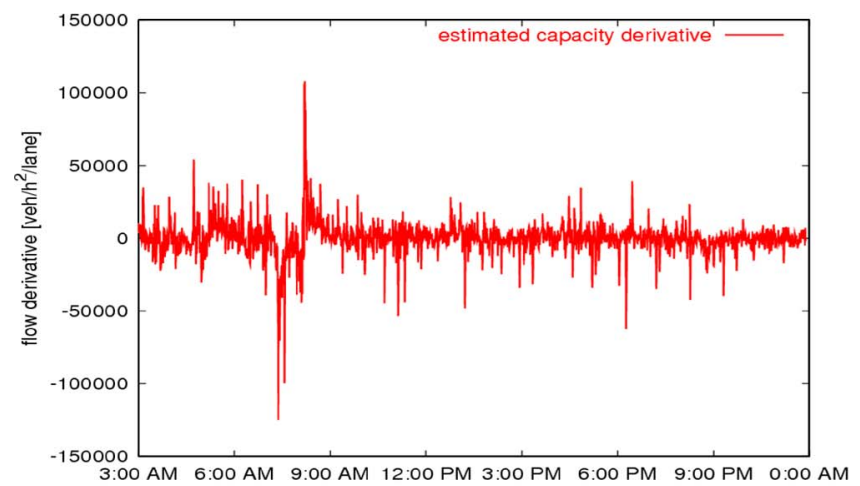

(g)



(b)

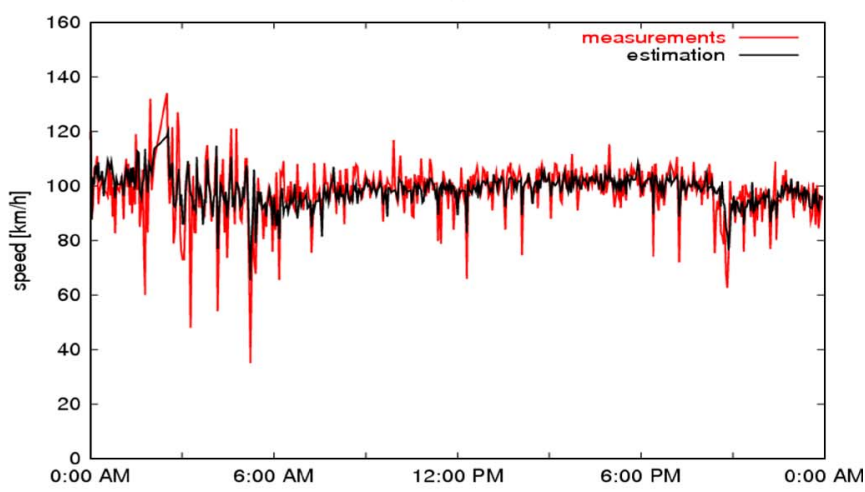

(d)

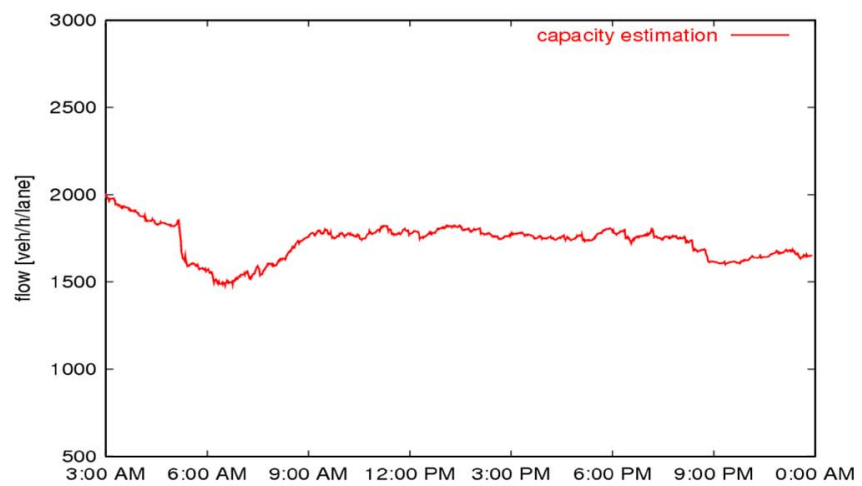

(f)

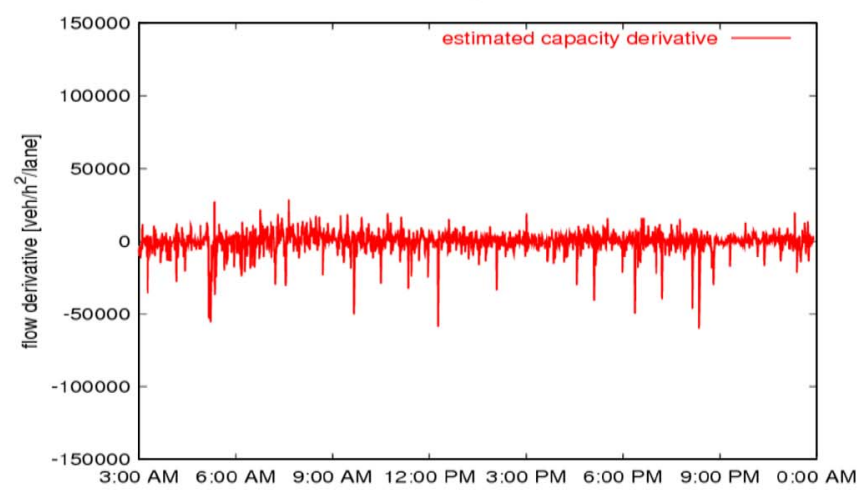

(h)

Fig. 12. Incident alarm results in the Naples direction on June 10, 2006. (a) and (b) At D11014. (c) and (d) At D11009. (e) and (f) Capacity estimates. (g) and (h) Estimated capacity derivatives.

RENAISSANCE is real-time applicable for a field site of the reported size (100 km in directed length). For more sizeable applications $(>150 \mathrm{~km})$, a decentralized approach to traffic surveillance may be needed.

\section{ACKNOWLEDGMENT}

The authors would like to thank the PON-SAM Project Manager L. Massa, Società Autostrade Meridionali (SAM), Naples, Italy. 


\section{REFERENCES}

[1] Y. Wang, M. Papageorgiou, and A. Messmer, "A real-time freeway network traffic surveillance tool," IEEE Trans. Control Syst. Technol., vol. 14, no. 1, pp. 18-32, Jan. 2006.

[2] Y. Wang, M. Papageorgiou, and A. Messmer, "RENAISSANCE—A unified macroscopic model-based approach to real-time freeway network traffic surveillance," Transp. Res. Part C, vol. 14, no. 3, pp. 190-212, Jun. 2006.

[3] M. W. Szeto and D. C. Gazis, "Application of Kalman filtering to the surveillance and control of traffic systems," Transp. Sci., vol. 6, no. 4, pp. 419-439, Nov. 1972.

[4] N. E. Nahi and A. N. Trivedi, "Recursive estimation of traffic variables: Section density and average speed," Transp. Sci., vol. 7, no. 3, pp. 269286, Aug. 1973.

[5] M. Papageorgiou, Application of Automatic Control Concepts to Traffic Flow Modeling and Control. New York: Springer-Verlag, 1983.

[6] M. Cremer and H. A. Schütt, "A comprehensive concept for simultaneous state observation, parameter estimation and incident detection," in Proc. 11th Int. Symp. Transp. Traffic Theory, Amsterdam, The Netherlands, 1990, pp. 95-111.

[7] R. R. Kohan and S. A. Bortoff, "An observer for highway traffic system," in Proc. 37th IEEE Conf. Decision Control, Tampa, FL, 1998, pp. 1012-1017.

[8] J. Meier and H. Wehlan, "Sectionwise modeling of traffic flow and its application in traffic state estimation," in Proc. 4th IEEE Conf. Intell. Transp. Syst., Oakland, CA, 2001, pp. 442-447.

[9] Y. Wang and M. Papageorgiou, "Real-time freeway traffic state estimation based on extended Kalman filter: A general approach," Transp. Res. Part B, vol. 39, no. 2, pp. 141-167, Feb. 2005.

[10] A. Hegyi, D. Girimonte, R. Babuska, and B. De Schutter, "A comparison of filter configurations for freeway traffic state estimation," in Proc. 9th Int. IEEE Conf. Intell. Transp. Syst., Toronto, ON, Canada, 2006, pp. 1029-1034.

[11] C. Antoniou, Online Calibration for Dynamic Traffic Assignment Models-Theory, Methods and Application. Saarbrïcken, Germany: VDM Verlag Dr. Müller, 2007.

[12] L. Mihaylova, R. Boel, and A. Hegyi, "Freeway traffic estimation within particle filtering framework," Automatica, vol. 43, no. 2, pp. 290-300, Feb. 2007.

[13] Y. Wang, M. Papageorgiou, and A. Messmer, "Real-time freeway traffic state estimation based on extended Kalman filter: A case study," Transp. Sci., vol. 41, no. 2, pp. 167-181, May 2007.

[14] D. Ngoduy, "Applicable filtering framework for online multiclass freeway network estimation," Phys. A, vol. 387, no. 2/3, pp. 599-616, Jan. 2008.

[15] Y. Wang, M. Papageorgiou, A. Messmer, P. Coppola, A. Tzimitsi, and A. Nuzzolo, "An adaptive freeway traffic state estimator," Automatica, vol. 45, no. 1, pp. 10-24, Jan. 2009.

[16] Y. Wang, M. Papageorgiou, and A. Messmer, "Real-time freeway traffic state estimation based on extended Kalman filter: Adaptive capabilities and real data testing," Transp. Res. Part A, vol. 42, no. 10, pp. 1340-1358, Dec. 2008

[17] H. M. Zhang, "Recursive prediction of traffic conditions with neural network models," J. Transp. Eng., vol. 126, no. 6, pp. 472-481, Nov./Dec. 2000.

[18] H. Chen and S. G. Muller, "Use of sequential learning for short-term traffic flow forecasting," Transp. Res. Part C, vol. 9, no. 5, pp. 319-336, Oct. 2001

[19] B. L. Smith, B. M. Williams, and R. K. Oswald, "Comparison of parametric and nonparametric models for traffic flow forecasting," Transp. Res. Part C, vol. 10, no. 4, pp. 303-321, Aug. 2002.

[20] E. I. Vlahogianni, M. G. Karlaftis, and J. C. Golias, "Optimized and metaoptimized neural networks for short-term traffic flow prediction: A genetic approach," Transp. Res. Part C, vol. 13, no. 3, pp. 211-234, Jun. 2005.

[21] C. Quek, M. Pasquier, and B. B. S. Lim, "POP-TRAFFIC: A novel fuzzy neural approach to road traffic analysis and prediction," IEEE Trans. Intell. Transp. Syst., vol. 7, no. 2, pp. 133-146, Jun. 2006.

[22] W. Zheng, D. Lee, and Q. Shi, "Short-term freeway traffic flow prediction: Bayesian combined neural network approach," J. Transp. Eng., vol. 132, no. 2, pp. 114-121, Feb. 2006.

[23] A. Karim and H. Adeli, "Fast automatic incident detection on urban and rural freeways using wavelet energy algorithm," J. Transp. Eng., vol. 129, no. 1, pp. 57-68, Jan./Feb. 2003.

[24] D. Srinivasan, X. Jin, and R. L. Cheu, "Evaluation of adaptive neural network models for freeway incident detection," IEEE Trans. Intell. Transp. Syst., vol. 5, no. 1, pp. 1-11, Mar. 2004.

[25] E. Tzamali, G. Akoumianakis, A. Argyros, and Y. J. Stephanedes, "Improved design for vision-based incident detection in transportation sys- tems using real-time view transformations," J. Transp. Eng., vol. 132, no. 11 , pp. 837-844, Nov. 2006.

[26] C. L. Mak and H. S. L. Fan, "Development of dual-station automated expressway incident detection algorithms," IEEE Trans. Intell. Transp. Syst., vol. 8, no. 3, pp. 480-490, Sep. 2007.

[27] M. Papageorgiou, J.-M. Blosseville, and H. Haj-Salem, "Modeling and real-time control of traffic flow on the southern part of Boulevard Périphérique in Paris-Part I: Modeling," Transp. Res. Part A, vol. 24, no. 5, pp. 345-359, Sep. 1990.

[28] A. Messmer and M. Papageorgiou, "METANET: A macroscopic simulation program for motorway networks," Traffic Eng. Control, vol. 31, no. 9, pp. 466-470, 1990

[29] A. Kotsialos, M. Papageorgiou, C. Diakaki, Y. Pavlis, and F. Middelham, "Traffic flow modeling of large-scale motorway networks using the macroscopic modeling tool METANET," IEEE Trans. Intell. Transp. Syst., vol. 3, no. 4, pp. 282-292, Dec. 2002.

[30] Y. Wang, M. Papageorgiou, and A. Messmer, "Algorithms and preliminary testing for traffic surveillance," Deliverable 3.2 for the Project RHYTHM (IST-2000-29427), Brussels, Belgium, Jul. 2003.

[31] Y. Wang, P. Coppola, A. Messmer, A. Tzimitsi, M. Papageorgiou, and A. Nuzzolo, "Extended field test of a real-time freeway network traffic state estimator," in Proc. Transp. Res. Board 86th Annu. Meeting (Paper CD-ROM), Washington, DC, 2007.



Yibing Wang (M'04) received the B.Sc. degree in electronics and computer engineering from Sichuan University, Sichuan, China, the M.Eng. degree in automatic control engineering from Chongqing University, Chongqing, and the Ph.D. degree in control theory and applications from Tsinghua University, Beijing, China.

He was with the Dynamic Systems and Simulation Laboratory, Department of Production Engineering and Management, Technical University of Crete, Chania, Greece, where he was a Postdoctoral Researcher from 1999 to 2001 and a Senior Research Fellow from 2001 to 2007. $\mathrm{He}$ is currently a Senior Lecturer with the Department of Civil Engineering, Monash University, Victoria, Australia. He is the Book Reviews Editor for Transportation Research Part C: Emerging Technologies. His research interests include traffic flow modeling, freeway traffic surveillance, ramp metering, route guidance, urban traffic signal control, and vehicular ad hoc networks. From 2000 to 2007, he participated in several European projects on freeway traffic surveillance and control and collaborated with transportation research and practice professionals from the U.K., Greece, Germany, Belgium, Italy, and the Netherlands.

Dr. Wang is an Associate Editor for the IEEE TRANSACTIONS ON INTELLigENT TRANSPORTATION SySTEMS. He is a member of the International Federation of Automatic Control (IFAC) Technical Committee on Transportation Systems and the Advisory Committee of the European Project Network of Excellence for Advanced Road Cooperative Traffic Management in the Information Society. He was the Vice Program Chair of the Ninth IEEE International Conference on Intelligent Transportation Systems (Toronto, Canada, 2006) and the Program Chair of the 13th IEEE International Conference on Intelligent Transportation Systems (Madeira Island, Portugal, 2010).

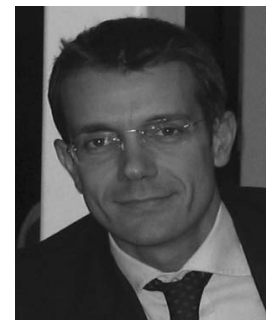

Pierluigi Coppola was born in Naples, Italy, in 1972. He received the M. Eng. degree in civil engineering and the Ph.D. degree in road infrastructures and transportation systems from the Federico II University of Naples.

$\mathrm{He}$ is currently an Assistant Professor of transports with the Department of Civil Engineering, "Tor Vergata" University of Rome, Rome, Italy. His research interests include advanced travelers information systems for road networks and public transportation systems, land use/transport interactions models, and network design methods. He is the author or a coauthor of more than 60 papers and book chapters on transportation system modeling. From 1998 to 2009, he was involved in European and national research projects and practiced his profession on travel demand forecasting, network design, and intelligent transportation systems in Italy and the Netherlands.

Dr. Coppola is a member of Program Committee of the European Transport Conference. 


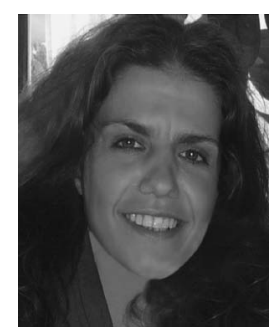

Athina Tzimitsi was born in Naoussa, Greece, in 1974. She received the Diploma degree in production engineering and management and the M.S. degree in operational research from the Technical University of Crete, Chania, Greece, in 2005 and 2007, respectively.

From 2004 to 2008, she was with the Dynamic Systems and Simulation Laboratory, Technical University of Crete. She is currently working in the private sector.

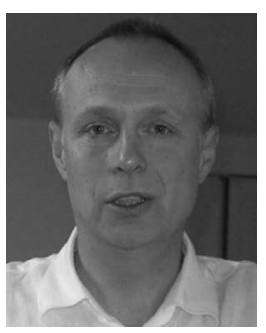

Albert Messmer received the Dipl.Ing. and Dr. Ing. degrees in electrical engineering (with a focus on control engineering) from the Technical University of Munich, München, Germany, in 1983 and 1994, respectively.

Since 1994, he has been an independent Consultant. He currently resides in Seeshaupt, Germany. Since 1983, he has been engaged in the modeling, simulation, optimization, and control of motorway networks and sewer systems. He has written more than 30 technical papers on the modeling and control of water flow and freeway traffic.

Dr. Messmer is a member of the Union of German Engineers.

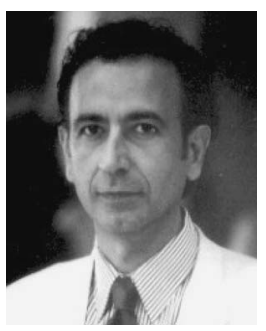

Markos Papageorgiou (F'99) was born in Thessaloniki, Greece, in 1953. He received the Dipl.-Ing. and Dr. Ing. (with honors) degrees in electrical engineering from the Technical University of Munich, München, Germany, in 1976 and 1981, respectively.

From 1976 to 1982, he was a Research and Teaching Assistant with the Control Engineering Chair, Technical University of Munich. He was a Free Associate with Dorsch Consult, Munich, from 1982 to 1988 and with the Institute National de Recherche sur les Transports et leur Sécurité, Arcueil, France, from 1986 to 1988. From 1988 to 1994, he was a Professor of automation with the Technical University of Munich. Since 1994, he has been a Professor with the Dynamic Systems and Simulation Laboratory, Technical University of Crete, Chania, Greece. He was a Visiting Professor with the Politecnico di Milano, Milano, Italy, in 1982, with the Ecole Nationale des Ponts et Chaussées, Paris, France, from 1985 to 1987, and with the Massachusetts Institute of Technology, Cambridge, in 1997 and 2000. He was a Visiting Scholar with the University of Minnesota, Minneapolis, in 1991 and 1993, the University of Southern California, Los Angeles, in 1993, and the University of California, Berkeley, in 1993, 1997, and 2000. He is the author of the books Applications of Automatic Control Concepts to Traffic Flow Modeling and Control (Springer, 1983) and Optimierung (Oldenbourg, 1991; 1996), the Editor of the Concise Encyclopedia of Traffic and Transportation Systems (Pergamon, 1991), a coauthor of Optimal Real-Time Control of Sewer Networks (Springer, 2005), and an Associate Editor for several journals. He is the author or a coauthor of some 300 technical papers. He is the Editorin-Chief of Transportation Research Part C. His research interests include automatic control and optimization theory and their applications to traffic and transportation systems, water systems, and further areas.

Dr. Papageorgiou is an Associate Editor for the IEEE Control Systems Society Conference Editorial Board. He also served as an Associate Editor for the IEEE TRANSACTIONS ON INTELLIGENT TRANSPORTATION SYSTEMS. He was the Chairman (1999-2005) and the Vice-Chairman (1994-1999) of the International Federation of Automatic Control Technical Committee on Transportation Systems. He received a Deutscher Akademischer Austausch Dienst scholarship (1971-1976), the 1983 Eugen-Hartmann Award from the Union of German Engineers, and a Fulbright Lecturing/Research Award (1997). He was the (first) recipient (2007) of the IEEE Outstanding Intelligent Transportation Systems Research Award.

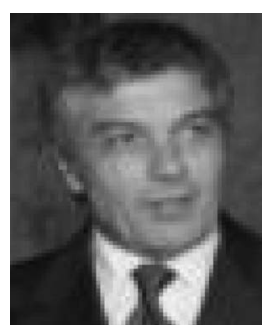

Agostino Nuzzolo was born in Calvi, Italy, in 1949. $\mathrm{He}$ received the M.Eng. degree in civil engineering from the Federico II University of Naples, Naples, Italy, in 1974.

He is currently a Full Professor of transportation planning with the Department of Civil Engineering, "Tor Vergata" University of Rome, Rome, Italy. His research interests include the theory of transportation systems and its application in transportation analysis, modeling, and planning. He is the author or a coauthor of four books on the schedule-based approach to dynamic transportation networks and some 150 technical papers and book chapters.

Prof. Nuzzolo is currently the President of the Italian Society of Lecturers of Transports. He has been responsible for national and international research projects on traffic and transportation planning, railway services pricing and design, master transport plans, and on technical-economic feasibility studies of transport infrastructures. 